\title{
Changing attitudes and behaviour towards bats by communities that live close to bat roosts after the 2013 Ebola viral disease outbreak in West Africa
}

\author{
Fidelia Ohemeng ${ }^{1}$, Elaine T Lawson ${ }^{2}$, Jesse S Ayivor ${ }^{2}$, Yaa Ntiamoa-Baidu ${ }^{3}$ \\ 1 Department of Sociology, University of Ghana, Legon, Accra, Ghana, ${ }^{2}$ Institute of Environment and Sanitation Studies, University of Ghana, Legon, \\ Accra, Ghana, ${ }^{3}$ Department of Animal Biology and Conservation Science, University of Ghana, Legon, Accra, Ghana \\ Keywords: global health
}

https://doi.org/10.29392/joghr.3.e2019037

Journal of Global Health Reports

Vol. 3, 2019

\begin{abstract}
Background
The 2013 Ebola virus disease (EVD) that hit parts of West Africa has been described as one of the largest outbreaks in recent decades. The outbreak was triggered by the spillover of pathogens from bats to human populations. In Ghana, bats pose a significant risk to humans because interactions between bats and human beings are common, and the henipahvirus and Ebola virus have been discovered in bats and in pigs. We investigated whether there had been a change in the way people who live close to bat roosts interact with bats in the wake of the 2013 EVD outbreak.
\end{abstract}

\section{Methods}

Questionnaires were distributed to 544 respondents drawn from five communities that live close to large bat roosts in southern Ghana. The data were analysed using descriptive statistics and $\chi^{2}$ tests.

\begin{abstract}
Results
The results show that a little over half of the respondents had changed the way they interact with bats as a result of the 2013 EVD outbreak. The reasons given by those who had not changed the way they interact with bats are that: EVD is not present in Ghana, bat meat when cooked properly pose no danger, and the belief that bats do not harbour viruses.
\end{abstract}

\section{Conclusions}

We conclude that public health education should be intensified to address the misconceptions that people still have about EVD.

Ebola virus disease (EVD) is a haemorrhagic disease with high fatality. The symptoms among others include fever, vomiting, and bleeding. Although there have been EVD outbreaks in the past four decades, the 2013 outbreak in parts of West Africa has been described as unprecedented both in scope and magnitude. ${ }^{1,2}$ Three countries in West Africa Guinea, Sierra Leone, and Liberia - were severely affected. During the 24 months of the outbreak, 28,625 Ebola cases were diagnosed, of which 11,325 people died yielding a case-fatality rate of $39.5 \% .^{1}$ There were reported cases across seven other nations on three continents. ${ }^{1,3}$ Considering the magnitude of the pandemic, the World Health Organisation (WHO) declared a Public Health Emergency of International Concern (PHEIC) on August 8, 2014. ${ }^{1}$ The outbreak devastated the already weak health systems and economies of the affected countries. ${ }^{4,5}$

It was reported that the patient zero in the 2013 EVD outbreak was a two-year old boy who came into contact with the droppings of bats. ${ }^{1,6}$ Apart from EVD, bats have been implicated in other diseases such as the Nipahvirus outbreak in Malaysia and Singapore in 1998 and 19997-9 and severe acute respiratory syndrome (SARS). The evidence so far indicates that the outbreaks occurred when people were exposed to the scratches, faecal droppings, urine, and the blood of bats. 6,8

Bats are very common in Ghana; large bat colonies have been found in several parts of the country including cities, towns and villages. For instance, bat colonies have been reported from Accra, Kumasi, Kyebi, Afram Plains, Ve Golokuati, Tanoboase and Oforikrom. ${ }^{10-13}$ The strawcoloured fruit bats (Eidolon helvum), epaulette fruit bats (Epomophorus gambianus) and hammer-headed bats (Hypsignathus-monstrous) are especially common. ${ }^{14}$ It is estimated that the Eidolon helvum that roosts at Accra and Tanoboase could contain over one and three million individuals respectively. ${ }^{15}$ In southern Ghana where bats are widely consumed, it is estimated that 128,000 of them are sold as bushmeat each year. ${ }^{10}$ The Techiman market in the 
Brong Ahafo region is noted for its bushmeat market where a wide range of wild animal carcasses, including bats, are sold. ${ }^{12}$ Bats are hunted also for ritualistic purposes. At Oforikrom and Buoyem, hunting of bats is part of their annual festival celebrations. ${ }^{12}$

In spite of the fact that human-bat interaction is common in Ghana, most Ghanaians do not associate bats with diseases. A study conducted before the 2013 Ebola outbreak showed that Ghanaians did not associate bats with diseases. ${ }^{13,14,16}$ At Accra and Tanoboase, where bats were widely hunted and consumed, participants in the Ohemeng et al. (14: 190), study intimated that they had been consuming bats for a very long time and that none of those who consumed bat meat had ever been sick from consuming bat meat. According to a participant: "if someone became sick after eating bat, then it means that person was already sick or about to get sick. I have not heard anything like that. No, not at all". The aim of this study therefore was to investigate whether there has been a change in the view that bats do not harbour diseases and how people interacted with bats had changed after the 2013 EVD outbreak. Though there has not been an outbreak of EVD in Ghana, bats still pose a significant risk to the population because the henipahvirus (both nipah and hendra virus) ${ }^{17,18}$ and Zaire EBOV (ZEBOV) ${ }^{15}$ have been discovered from a colony of Eidolon helvum in Accra, and also from pigs in Ve Golokuati, suggesting a transference from bats to animals. ${ }^{15}$ This means that a jump of the virus from bats and livestock to humans is possible.

\section{METHODS}

\section{THE STUDY COMMUNITIES}

Five communities from southern Ghana were selected for the study. These are Tanobaose in the Brong Ahafo region, Ve-Golokuati, Kpeve and Gbefi-Tornu in the Volta region and Accra in the Greater Accra region. These communities were chosen because they had large bat roosts. Please refer to Ohemeng et al. ${ }^{14}$ and Ayivor et al. ${ }^{16}$ for detailed description of Tanobaose, Accra and Ve Golokuati. An interesting story was told about the origins of the bats at the 37 Military Hospital. According to the respondents, the bats accompanied a chief from the Eastern Region who was admitted at the hospital. When the chief died the corpse was removed at night as is the custom when a chief dies. The bats, not knowing that the chief had died and had been removed (because bats feed at night and return to roost in the day) have remained there ever since waiting for their chief. At Gbefi-Tornu, thousands of Epomophorus gambianus roost in mango trees located in people's homes. Similarly, Kpeve hosts thousands of Eidolon helvum, which roost in huge silk cotton trees (Ceiba pentandra) within the community.

\section{DATA COLLECTING}

The study employed the survey design in which participants answered questions during a single interview. The sampling method used was based on purposive sampling. Communities with large bat colonies were identified in southern Ghana. We then selected five of them from the various ecological zones and also on rural or urban types. Tanoboase is rural and also in the transition zone, Ve-Golokuate, Kpeve and Gbefi-Tornu are also rural and in the Volta representing the forest and transition and Accra is urban and represents the coastal belt. The systematic random sampling was used in four of the study site with the exception of Accra. At Accra, participants were purposively recruited from workers at the 37 Military Hospital, the Department of Parks and Gardens and the Military Barracks (the bat roosts are located here) and not from the larger population. The criteria for inclusion were that participants should be 18 years and above and must be willing to take part in the study. Participants were approached and the study explained to them. Those who agreed to take part were then interviewed faceto-face. Thus, the recruitment rate was $100 \%$. The instrument used to collect the data were closed and open-ended questionnaire and included questions on the demographic characteristics of participants, uses of bats, risk and exposure of participants to bats, bat conservation and management, risk communication messages, and changes in behaviour towards bats.

The data were coded and analysed using the IBM Statistical Package for the Social Sciences (SPSS) version 21 (IBM Corporation, Armonk, New York, USA). Descriptive statistics and simple frequencies were used to analyse the data. A $\chi^{2}$ test was conducted to test the statistical significance of changes in the behaviour of participants.

\section{ETHICAL CONSIDERATION}

Ethical clearance was obtained from the Institutional Review Board (IRB) of the Noguchi Memorial Institute for Medical Health at the University of Ghana. The ethical clearance number is Study Number 002/13/14, Internal Number 1796. Consent was sought from all the respondents before the questionnaires were administered. The respondents who were literate signed the consent forms whiles the non-literates were made to thumb-print.

\section{RESULTS}

\section{DEMOGRAPHIC CHARACTERISTICS OF RESPONDENTS}

In all 554 participants took part in the survey. This included 148 from Accra, 163 from Tanoboase, 125 from Ve-Golokuate, 72 from Kpeve and 46 from Gbefi-Tornu. Females constituted $46.4 \%$ and males $53.6 \%$ of the sample. The participants were aged between 18 and 55 years. On the level of education of respondents, $16.2 \%$ had primary/middle school level education, 35.1\% Junior High School education, 22.0\% Senior High School level education, and 8.4\% with tertiary level education. With respect to religious affiliation, majority of the respondents (88\%) were Christians, while $7.7 \%$ were Muslims (Table 1). The main occupation of participants was farming (34\%) and trading (17.3\%), reflecting the fact that the communities were all rural except Accra. Occupations designated under 'other' constituted $19.1 \%$, and this included military personnel, teachers, gardeners, and midwives, most of whom worked in Accra. 
Table 1. Demographic characteristics of respondents

\begin{tabular}{|c|c|c|}
\hline Variable & Frequency & $\%$ \\
\hline \multicolumn{3}{|l|}{ Community: } \\
\hline Accra & 143 & 26.7 \\
\hline Tanoboase & 163 & 29.4 \\
\hline Ve-Golokuati & 125 & 22.6 \\
\hline Kpeve & 72 & 13 \\
\hline Gbefi & 46 & 8.3 \\
\hline Total & 554 & \\
\hline \multicolumn{3}{|l|}{ Sex: } \\
\hline Male & 291 & 53.6 \\
\hline Female & 252 & 46.4 \\
\hline Total & 543 & \\
\hline \multicolumn{3}{|l|}{ Age: } \\
\hline$<15$ & 27 & 5 \\
\hline $16-25$ & 105 & 19.3 \\
\hline $26-35$ & 121 & 22.3 \\
\hline $36-45$ & 100 & 18.4 \\
\hline $46-55$ & 103 & 19.0 \\
\hline$>56$ & 87 & 16.0 \\
\hline Total & 543 & \\
\hline \multicolumn{3}{|l|}{ Educational level: } \\
\hline No Education & 89 & 18.3 \\
\hline Primary/Middle School & 79 & 16.2 \\
\hline JHS & 171 & 35.1 \\
\hline SHS & 107 & 22.0 \\
\hline Tertiary & 41 & 8.4 \\
\hline Total & 487 & \\
\hline \multicolumn{3}{|l|}{ Religion: } \\
\hline Christian & 468 & 88.0 \\
\hline Muslim & 41 & 7.7 \\
\hline Traditional religion & 18 & 3.4 \\
\hline Other & 5 & 0.9 \\
\hline Total & 532 & \\
\hline
\end{tabular}

JHS - Junior High School, SHS - Senior High School

\section{CONTACT WITH BATS}

Since the communities studied were close in proximity to bat roost, we wanted to know whether the participants came into direct contact with bats and the nature of the contact. The findings show that some of the participants came into direct contact with the bats. This was mainly through hunting, consumption, and the droppings of bats. More than a third (38.1\%) of the respondents specified that they consumed bat meat, while over two-thirds (61.9\%) did not consume bats. The main method of preparation was boiling or roasting (65.5\%), and frying (9.0\%). About 36.6\% indicated that they killed the bats themselves, $31.7 \%$ obtained it from hunters, and $11.0 \%$ said they bought it from the market. About 20.7\% indicated that they obtained it from food vendors, on their farms, and household meals. The weapons used to hunt the bats included guns, sticks, nets, and catapults. As is consistent with other studies, ${ }^{13,14}$ men were more inclined to consume bats more than women. While $68.6 \%$ of the men consumed bat meat, only $31.4 \%$ of women did. Furthermore, older respondents were more inclined to consume bat meat more than younger respondents. More respondents between the ages of 46 to 55 (20.8\%) were found to consume bats more than people from other age groups; this was followed by respondents in the 36-45 age bracket (19.8\%).

\section{PERCEPTION THAT BATS CAN CAUSE DISEASES}

We wanted to find out whether participants thought bats harbor diseases. The majority of the respondents, $74.6 \%$ of the respondents indicated that bats can be the source of diseases, while $25.4 \%$ did not think that bats carry diseases. Of the participants who responded in the affirmative, $45.7 \%$ (177) were females and 54.3\% (210) were males. Additionally, $18.2 \%$ (64) of the participants with no formal education mentioned that bats harbor diseases, and 9.4\% of participants with tertiary education indicated yes to the question. Of the participants who indicated that they consume bats (201) $67.2 \%$, that is 135 mentioned that there is a link between bats and diseases, whiles $32.8 \%$ (66) did not think that there is an association between bats and diseases. On the other hand, of those who said bats carry diseases, $35.2 \%$ consumed bats and $74.4 \%$ did not consume (Table 2). We did not find any statistical significant differences in the responses among the various communities $\left(\chi^{2}{ }_{4}\right.$ $=4.831, P=0.31$ ). About the type of diseases bats may carry, majority of them indicated Ebola; while others mentioned rabies, malaria, tetanus and typhoid fever.

\section{KNOWLEDGE OF EBOLA}

As indicated above, the 2013 EVD outbreak in parts of West Africa was of global proportions, so we tested the knowledge of respondents on EVD. Knowledge on EVD was near universal, with $95.9 \%$ indicating that they had heard of EVD. The mode of transmission as indicated by the participants included bites and consumption of bats, and other bushmeat such as monkeys, cane rat (grasscutter/Thyronomys swinderianus). Others also mentioned that EVD is airborne and could be transmitted when an individual comes into contact with an infected person. The source of knowledge was through the media, family, friends, and educational institutions. In the media, the radio was the major source of information for most of the participants (86.2\%).

\section{CHANGES IN INTERACTION WITH BATS}

One of the objectives of this study was to investigate whether there have been changes in the way people interact with bats (hunting, consuming, processing) in the two years since the EVD outbreak in parts of West Africa. Of the participants studied, $51.2 \%$ that is 261 indicated that they had changed the way they interact with bats, whiles $48.8 \%$, (248) indicated that they had not changed the way they interacted with bats. However, a $\chi-2$ test indicates that the change in interaction was not statistically significant $\left(\chi^{2}=0.279, \mathrm{~N}=516, P=0.597\right)$. Of the 261 participants who 
Table 2. Demographic characteristics and perception that bats cause diseases

\begin{tabular}{|c|c|c|c|c|c|}
\hline \multirow{2}{*}{$\begin{array}{l}\text { Variables } \\
\text { Name of community }\end{array}$} & \multicolumn{2}{|c|}{ Yes } & \multicolumn{2}{|c|}{ No } & \multirow[b]{2}{*}{ Total } \\
\hline & $\%$ & $\mathrm{~N}$ & $\%$ & $N$ & \\
\hline Accra & 23.8 & 93 & 27.8 & 37 & 130 \\
\hline Tanoboase & 28.9 & 113 & 35.3 & 47 & 160 \\
\hline Ve Golokuati & 25.1 & 98 & 24 & 18 & 122 \\
\hline Kpeve & 13.6 & 53 & 10.5 & 14 & 67 \\
\hline Gbefi-Tornu & 8.7 & 34 & 8.3 & 11 & 45 \\
\hline \multicolumn{6}{|l|}{ Sex: } \\
\hline Female & 45.7 & 177 & 48.9 & 64 & 241 \\
\hline Male & 54.3 & 210 & 51.1 & 67 & 277 \\
\hline \multicolumn{6}{|l|}{ Educational level: } \\
\hline No Formal & 18.2 & 64 & 2.9 & 24 & 88 \\
\hline Primary & 18.2 & 64 & 9.6 & 11 & 75 \\
\hline JHS & 34.5 & 121 & 38.3 & 44 & 165 \\
\hline SHS & 19.7 & 69 & 27.0 & 31 & 100 \\
\hline Tertiary & 9.4 & 33 & 4.3 & 5 & 38 \\
\hline \multicolumn{6}{|l|}{ Consumption of bats: } \\
\hline Yes & 35.2 & 135 & 50.0 & 66 & 201 \\
\hline No & 64.8 & 249 & 50.0 & 66 & 315 \\
\hline
\end{tabular}

JHS - Junior High School, SHS - Senior High School

Table 3. Demographic characteristics and change in interaction

\begin{tabular}{lcccccc}
\hline Community & \multicolumn{2}{c}{ Yes } & & & No & Total \\
\hline & $\%$ & $\mathrm{~N}$ & $\%$ & $\mathrm{~N}$ & \\
Accra & 47.3 & 62 & 52.7 & 69 & 131 \\
Tanoboase & 64.6 & 104 & 35.4 & 54 & 161 & 119 \\
Ve Golokuati & 56.3 & 67 & 43.7 & 52 & 62 \\
Kpeve & 38.7 & 24 & 61.3 & 38 & 36 \\
Gbefi-Tornu & 16.3 & 7 & 83.7 & 36 & 43 \\
\hline
\end{tabular}

said they had changed their behaviour in the last two years, $46.7 \%$ are females and $53.3 \%$ are males. Furthermore, $21.5 \%$ of participants with no formal education mentioned they had changed the way they interact with bats, while $19.0 \%$ primary school leavers, 32.1\% JHS, 20.7\% SHS, and $6.8 \%$ had tertiary level education also indicated same. Of the 201 participants who indicated that they eat bat meat, $40 \%$ indicated that they had stopped consuming bats, whiles $36.1 \%$ said they had not stopped bat meat consumption. The change in interaction with bats was similar across all age groups. In the 15-25 age group $21.2 \%$ had changed their interaction while $19.0 \%$ had not, in the $46-55$ age group $20.5 \%$ had changed and $18.5 \%$ had not changed, and in the 55 and above age group, $16.6 \%$ had changed and $15.3 \%$ had not changed. On the other hand, in the 26-35 age group $18.9 \%$ had changed whiles $23.4 \%$ had not changed. Likewise, in the $36-45$ age group; $18.1 \%$ had changed and $19.4 \%$ had not changed. Changes were also observed in some of the communities. Whiles changes were observed in Accra, Tanoboase, and Ve Golokuati, no changes were observed in Kpeve and Gbefi-Tornu (Table 3).
Of those who indicated they had changed how they interact with bats in the last two years, $78 \%$ cited the fear of being infected with Ebola as the main reason for the change. On the contrary, of those who indicated that they had not changed how they interact with bats, $23.3 \%$ maintained that bats do not carry any disease, $4.8 \%$ intimated that bats pose no danger when properly cooked, $4.1 \%$ said Ebola was not yet in Ghana and another $4.1 \%$ disclosed that bat meat was scarce that was why they had stopped consumption. Other reasons given were religious beliefs, that they (respondents) are used to the bats and have put in place safety measures. It is worthy to note that $32.2 \%$ of those who indicated that they had not changed their behavior said they rarely encounter bats and $18.5 \%$ said they have never consumed bat meat (Table 4).

\section{DISCUSSION}

The aim of this paper was to find out whether the risk perception about bats have changed after a major EVD outbreak in neighbouring countries in 2013. The findings in- 
Table 4. Reasons for change in interaction with bats

\begin{tabular}{lccc}
\hline & \multicolumn{1}{c}{ Have your behaviour changed? } & \\
\hline Reasons for change & Yes & No & Total \\
Fear of contracting Ebola/carry disease & $193(78.8 \%)$ & $6(4.1 \%)$ & $199(50.9 \%)$ \\
Bats carry no disease & $4(1.6 \%)$ & $34(23.3 \%)$ & $38(9.7 \%)$ \\
Proper cooking makes it safe & $0(0.0 \%)$ & $7(4.8 \%)$ & $7(1.8 \%)$ \\
Religion & $2(0.8 \%)$ & $3(2.1 \%)$ & $5(1.3 \%)$ \\
No Ebola in Ghana & $0(0.0 \%)$ & $6(4.1 \%)$ & $6(1.5 \%)$ \\
Safety measures taken & $0(0.0 \%)$ & $2(1.4 \%)$ & $2(0.5 \%)$ \\
Rarely come into contact with them & $8(3.3 \%)$ & $47(32.2 \%)$ & $55(14.1 \%)$ \\
Do not eat them & $6(2.4 \%)$ & $27(18.5 \%)$ & $33(8.4 \%)$ \\
Scarcity of bats & $6(2.4 \%)$ & $6(4.1 \%)$ & $12(3.1 \%)$ \\
Used to them now & $26(10.6 \%)$ & $7(4.8 \%)$ & $33(8.4 \%)$ \\
Total & 245 & 145 & 390
\end{tabular}

dicate that knowledge about EVD was near universal, with 95\% of the respondents indicating that they had heard about Ebola. Furthermore, participants exhibited the right knowledge about the mode of transmission of EVD. They indicated that EVD is transmitted through contact with infected bats, monkeys and cane rat, and through direct contact with infected persons. This corroborates research conducted by Adongo and colleagues ${ }^{19}$ and Gbogbo and Kyei ${ }^{20}$ on the knowledge about EVD. They found that awareness and knowledge about EVD was high among the participants studied. A similar study conducted in Nigeria also showed a high level of awareness and mode of transmission among study participants. ${ }^{21}$ This is because the media's reportage on the outbreak was extensive, though no EVD was recorded Ghana. In addition, Ghana was selected as the command centre from where the international community sent aid to the affected countries. Some Ghanaians also joined volunteers in the effort. No wonder the findings show that majority of the participants identified radio as the source of information and knowledge about EVD.

Further, the extensiveness of the public health campaign influenced the risk perception of the majority of the participants who indicated that bats could cause diseases. The diseases mentioned by the participants included EVD, rabies, malaria, tetanus and typhoid. This is in sharp contrast to a previous study conducted in three of the study areas-Tanoboase, Ve Golokuati and Accra-where majority of the participants did not associate bats with diseases. ${ }^{13,14}$ In that study, one of the reason participants gave for their perception was that they had been consuming bat meat for a long time and yet nobody had been taken ill.

Changes in behaviour has proved to be useful in containing and preventing infectious diseases. Rubin GJ, Amlôt R, Page $L$ and Wessely $S^{22}$ are of the view that in the event of a disease outbreak, people are more likely to change their behaviour if they perceive that the recommended behaviour is helpful, that there is the high likelihood that they may be affected, that the illness has severe consequences, and that the illness is difficult to treat. Behavioural changes also result when the level of anxiety and worry is high and people perceive that the government is providing clear and sufficient information. In this study, more than half of the par- ticipants changed their behaviour because they perceived that there was a high likelihood of them being affected and were aware of the devastation caused by the disease in the affected countries. A substantial number of participants (78.8\%) who stated they have changed the way they interact with bats cited the fear of being infected with Ebola as the reason for a change in behaviour.

While a little over half of the participants had altered their behaviour since the 2013 Ebola outbreak, 48.8\% had not made changes to their behaviour. Of that number, $23.3 \%$ still did not belief that bats can cause diseases, and others also indicated that cooking properly the meat would kill any viruses if any, and still others thought that EVD was not yet in Ghana. A major challenge in public health campaign is that high knowledge about a disease normally do not correspond to behaviour change. As Rubin and Amlôt ${ }^{22}$ observed, convincing people that a threat is real and therefore they should change their behaviour could be difficult. In their study on the perception and change in behaviour after the 2009 swine flu outbreak, they found a limited change in behaviour among study participants. Most of their participants had not changed the frequency of hand washing, disinfecting and had not discussed plans with a "flu friend". Furthermore, misconceptions about new and emerging diseases can also contribute to people not changing their behaviour. In a study on the perception of EVD in Ghana, Adongo PB, Tabong PT- ${ }^{19}$ found that there were misconceptions among study participants on the causes of EVD. Some of the misconceptions they detailed were that EVD was spiritually caused, was transmitted through mosquito bites and was airborne. Though misconception of EVD did not feature in our study, it could be a contributing factor to people not changing how they interact with bats.

\section{CONCLUSIONS}

This study set out to explore the changes in behaviour among people who live close to bat roost after the 2013 Ebola outbreak in parts of West Africa. The findings indicate that knowledge about the EVD was very high among the participants. Though some of the participants had made changes to the way they interact with bats, a sizeable num- 
ber had not. This is due to participants' belief that they did not think they were at a high risk. The findings show that there is still a lot of public health education to be done about the mode of transmission of Ebola viral disease to deal with misconceptions. The education should be detailed, drawing on the culture and beliefs of the people, and it must also be continuous whether there is a disease outbreak or not.

The limitation of the study is that the sample size is small to allow for generalizability of the findings. In addition, the study areas do not reflect all of the features (culture, religion, etc) of Ghana. Thus, the findings of the study cannot be generalised to the rest of the country and beyond.

\section{CORRESPONDENCE TO:}

Fidelia Ohemeng

Department of Sociology

University of Ghana

P. O. Box LG 65

Accra

Ghana

fohemeng@ug.edu.gh 


\section{REFERENCES}

1. Shultz JM, Espinel Z, Espinola M, Rechkemmer A. Distinguishing epidemiological features of the 2013-2016 West Africa Ebola virus disease outbreak. Disaster Health. 2016;3(3):78-88. doi:10.1080/216650 $\underline{44.2016 .1228326}$

2. Shultz JM, Cooper JL, Baingana F, et al. The role of fear-related behaviors in the 2013-2016 West Africa Ebola virus disease outbreak. Curr Psychiatry Rep. 2016;18(11):104. doi:10.1007/s11920-016-0741-y

3. Rübsamen N, Castell S, Horn J, et al. Ebola risk perception in Germany, 2014. Emerg Infect Dis. 2015;21(6):1012-1018. doi:10.3201/eid2106.150013

4. Youde J. The ebola outbreak in Guinea, Liberia, and Sierra Leone. E-International Relations. 2014;26.

5. Buseh AG, Stevens PE, Bromberg M, Kelber ST. The Ebola epidemic in West Africa: challenges, opportunities, and policy priority areas. Nurs Outlook. 2015;63:30-40. doi:10.1016/j.outlook.2014.12.013

6. Baize S, Pannetier D, Oestereich L, et al. Emergence of Zaire Ebola virus disease in Guinea. $N$ Engl J Med. 2014;371(15):1418-1425. doi:10.1056/nej moa1404505

7. Looi LM, Chua KB. Lessons from the Nipah virus outbreak in Malaysia. Malays J Pathol. 2007;29:63-67.

8. Luby SP, Hossain MJ, Gurley ES, et al. Recurrent zoonotic transmission of Nipah virus into humans, Bangladesh, 2001-2007. Emerg Infect Dis. 2009;15(8):1229-1235. doi:10.3201/eid1508.081237

9. Pigott DM, Golding N, Mylne A, et al. Mapping the zoonotic niche of Ebola virus disease in Africa. eLife. 2014;3:e04395. doi:10.7554/elife.04395

10. Kamins AO, Restif O, Ntiamoa-Baidu Y, et al. Uncovering the fruit bat bushmeat commodity chain and the true extent of fruit bat hunting in Ghana, West Africa. Biol Conserv. 2011;144(12):3000-3008. do i:10.1016/i.biocon.2011.09.003

11. Hayman DTS, Wang LF, Barr J, et al. Antibodies to henipavirus or henipa-like viruses in domestic pigs in Ghana, West Africa. PLoS One. 2011;6(9):e25256. do i:10.1371/journal.pone.0025256

12. Anti P, Owusu M, Agbenyega O, et al. Human-bat interactions in rural West Africa. Emerg Infect Dis. 2015;21(8):1418-1421. doi:10.3201/eid2108.142015
13. Lawson ET, Ayivor JS, Ohemeng F, Ntiamoa-Baidu Y. Social determinants of a potential spillover of batborne viruses to humans in Ghana. Int J Bio. 2016;8(2):66. doi:10.5539/ijb.v8n2p66

14. Ohemeng F, Lawson ET, Ayivor J, Leach M, Waldman L, Ntiamoa-Baidu Y. Socio-cultural Determinants of Human-Bat Interactions in Rural Ghana. Anthrozoös. 2017;30(2):181-194. doi:10.1080/ $\underline{08927936.2017 .1310984}$

15. Hayman DTS, Yu M, Crameri G, et al. Ebola virus antibodies in fruit bats, Ghana, West Africa. Emerg Infect Dis. 2012;18(7):1207-1209. doi:10.3201/eid180 $\underline{7.111654}$

16. Ayivor JS, Ohemeng F, Tweneboah Lawson E, Waldman L, Leach M, Ntiamoa-Baidu Y. Living with bats: The case of Ve Golokuati Township in the Volta Region of Ghana. J Environ Public Health. 2017;2017:1-11. doi:10.1155/2017/5938934

17. Hayman DTS, Suu-Ire R, Breed AC, et al. Evidence of henipavirus infection in West African fruit bats. PLoS One. 2008;3(7):e2739. doi:10.1371/journal.pon e.0002739

18. Baker KS, Todd S, Marsh GA, et al. Novel, potentially zoonotic paramyxoviruses from the African straw-colored fruit bat Eidolon helvum. $J$ Virol. 2013;87(3):1348-1358. doi:10.1128/jvi.01202-1 $\underline{2}$

19. Adongo PB, Tabong PTN, Asampong E, Ansong J, Robalo M, Adanu RM. Beyond Knowledge and Awareness: Addressing Misconceptions in Ghana's Preparation towards an Outbreak of Ebola Virus Disease. PLoS One. 2016;11(2):e0149627. doi:10.1371/ journal.pone.0149627

20. Gbogbo F, Kyei MO. Knowledge, perceptions and attitude of a community living around a colony of straw-coloured fruit bats (Eidolon helvum) in Ghana after Ebola virus disease outbreak in West Africa. Zoonoses Public Health. 2017;64(8):628-635. doi:10.11 11/zph.12357

21. Gidado S, Oladimeji AM, Roberts AA, Nguku P, Nwangwu IG, Waziri NE. Public knowledge, perception and source of information on Ebola virus disease-Lagos, Nigeria; September, 2014. PLoS Curr. 2015;7:ecurrents.outbreaks.0b805cac244d700a47d6a3713ef2d6db. 
22. Rubin GJ, Amlôt R, Page L, Wessely S. Public perceptions, anxiety, and behaviour change in relation to the swine flu outbreak: cross sectional telephone survey. BMJ. 2009;339:b2651. doi:10.1136/ bmj.b2651 